\title{
From Expected Propagator Distribution to Optimal Q-space Sample Metric
}

\author{
Hans Knutsson ${ }^{1}$ and Carl-Fredrik Westin $^{2}$ \\ 1 Linköping University, Sweden \\ 2 Harvard Medical School, USA
}

\begin{abstract}
We present a novel approach to determine a local q-space metric that is optimal from an information theoretic perspective with respect to the expected signal statistics. It should be noted that the approach does not attempt to optimize the quality of a pre-defined mathematical representation, the estimator. In contrast, our suggestion aims at obtaining the maximum amount of information without enforcing a particular feature representation.

Results for three significantly different average propagator distributions are presented. The results show that the optimal q-space metric has a strong dependence on the assumed distribution in the targeted tissue. In many practical cases educated guesses can be made regarding the average propagator distribution present. In such cases the presented analysis can produce a metric that is optimal with respect to this distribution. The metric will be different at different q-space locations and is defined by the amount of additional information that is obtained when adding a second sample at a given offset from a first sample. The intention is to use the obtained metric as a guide for the generation of specific efficient q-space sample distributions for the targeted tissue.
\end{abstract}

\section{Introduction}

The discussion concerning optimal q-space sampling strategies has been lively from the very start of diffusion imaging and is continuing to be a major topic of research [1] - 10]. Existing sampling schemes are based on experience combined with more or less ad hoc approaches of which many display interesting features. There is, however, no consensus regarding the choice of q-sample distribution in any given situation. Here we try to improve this situation by introducing a novel approach to determine a local q-space metric that is optimal from an information theoretic perspective with respect to the expected signal statistics.

The metric will be dependent on the q-space location an indicates the information gain, as a function of distance and direction, when adding a sample in a second q-space location. The obtained metric can then serve as a guide for the generation of specific q-space sample distributions e.q. sample distributions obtained in the manner described in [10. It should be noted that the approach differs significantly from the classical estimation theory approach, e.g. one based on Cramer-Rao bounds [12. The latter requires a pre defined mathematical

P. Golland et al. (Eds.): MICCAI 2014, Part III, LNCS 8675, pp. 217-224, 2014.

(C) Springer International Publishing Switzerland 2014 
representation, the estimator. Our suggestion aims at obtaining the maximum amount of information without enforcing a particular feature representation.

\section{Theory}

The mutual information (originally termed rate of transmission) between to signals relates directly to the entropies involved and can be estimated from the joint signal statistics [1]. Using a Gaussian signal+noise source model, which is a quite reasonable starting point in the present context, the estimate is directly related to the canonical correlation between two signals, a and b, and is given in bits by:

$$
I_{a b}=-\frac{1}{2} \log _{2}\left(\frac{\left[\mathbf{C}_{a a}|| \mathbf{C}_{b b} \mid\right.}{|\mathbf{C}|}\right) \quad \text { where } \quad \mathbf{C}=\left(\begin{array}{ll}
\mathbf{C}_{a a} & \mathbf{C}_{a b} \\
\mathbf{C}_{a b} & \mathbf{C}_{b b}
\end{array}\right)
$$

and $\mathbf{C}$.. are covariance matrices. For the one-dimensional case this reduces to: $I_{a b}=-\frac{1}{2} \log _{2}\left(1-\rho_{a b}^{2}\right)$, where $\rho_{a b}$ is the correlation between the two variables. This expression can also be used to estimate the information from a single signal by measuring the correlation between the signal with and added noise realization and the same signal without noise. In order to obtain an estimate of a local information based q-space metric we can compute the information gain, $I_{\Delta}$, from measuring in a second q-space location, $q_{b}$, given that we already have a measure at a first location, $q_{a} . I_{\Delta}$ is obtained as the information due to the second measurement alone minus the mutual information between the two measured signals, i.e. the information that is already present due to the first measurement:

$$
I_{\Delta}=I_{b_{0} b}-I_{a b}=\frac{1}{2} \log _{2}\left(\frac{1-\rho_{a b}^{2}}{1-\rho_{b_{0} b}^{2}}\right)
$$

In this expression $b_{0}$ is the true, noise free, source signal at the second location. It can be noted that equation (2) will give $I_{\Delta}=\frac{1}{2} \log _{2}\left(\frac{2 \mathrm{SNR}+1}{\mathrm{SNR}+1}\right)$ if the second measurement is taken at the same location as the first. For reasonably high SNR (signal to noise ratio) this corresponds 0.5 bits or, equivalently, improving measurement SNR by $\sqrt{2}(3 \mathrm{~dB})$.

It should also be noted that the Gaussian and additive assumptions are not crucial since mutual information between two variables is a monotonically increasing function of the correlation even in highly non-Gaussian and non-additive cases 13 .

\section{Method}

To obtain the statistics of the q-space signals we generate a large number of q-space response examples. Using these examples correlation estimates between any two q-space locations, as well as correlations between different instances of 
the same location, can be estimated. From these correlations the added information from measuring in a second q-space location, given a first measurement in any other location, can be found. The fact that each voxel in will contain a huge number of different propagators determining the q-space signals, and that a substantial intra voxel variation in propagator size and shape can be expected, makes it natural to use a Gaussian as a first approximation of the q-space response magnitude.
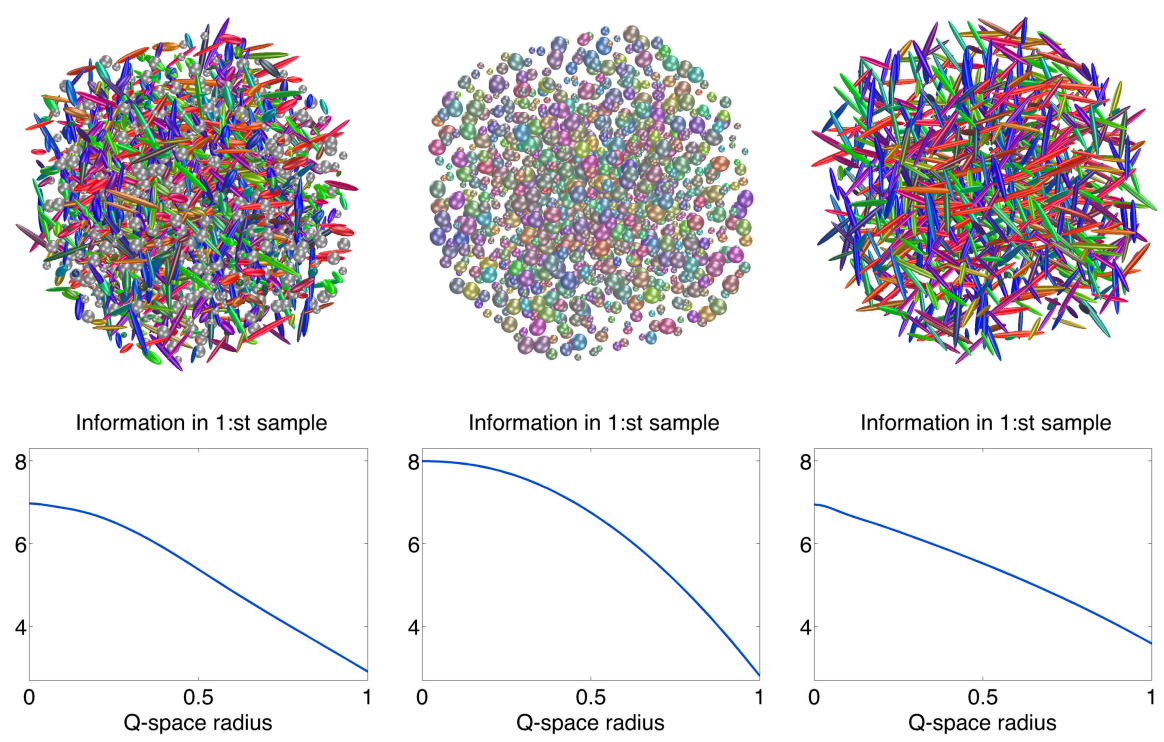

Fig. 1. The upper row illustrates three archetypal average propagator distributions. The plots show iso-surfaces of the Gaussian examples with centers distributed evenly in a volume of tissue. From left to right is shown: Allsorts - Varying in orientation, shape and size (Left). Round - Almost spherical propagators of varying in size (Center). Stick - Highly anisotropic only varying in orientation (Right). For each distribution the bottom row shows $I_{a_{0} a}(\|\mathbf{q}\|)$, the amount of information that is given by the first sample at a given radius in q-space.

The example generator was set to produce 3D Gaussian q-space responses having one long axis and two equal short axes. All generated distributions had 300 different long axes orientations evenly distributed to cover all 3D orientations. The size of the average propagators was also varied. The total number of the propagator examples of a given 'tissue volume' was set to vary as the inverse of the volume, i.e. the total volume of the smaller propagators was equal to the total volume of the larger propagators. The average size of the propagators was set to vary logarithmically in the specified range. The ratio between the long and short axes was also set to vary logarithmically in the specified range while keeping the propagator volume constant. The intention is to study a few archetypal situations that can be easily understood, not to mimic real tissue. A 
multitude of approaches for modelling biological tissue have been put forward and such models can readily be incorporated in the present framework. Doing so is, however, beyond the scope of this paper.

Listed below and shown in figure 1 are the three different distributions generated to study the effects different average diffusion propagator distributions will have on the q-space sampling metric. All three generated distributions are rotation invariant. Also note that the individual spatial propagator positions are not important for our analysis.

Allsorts - Long/short axis ratios from 1 to 10 and volumes from 0.5 to 2 .

Round - A long/short axis ratio of 1.15 and volumes from 0.22 to 4.5.

Stick - A long/short axis ratio of 10 and volumes from 0.5 to 2 .

The lower part of figure 1 shows the information given by the first sample as a function of $\mathrm{q}$-space radius. The q-space radius refered to in the present work is a relative entity, the relation to physical q-space depends on scanner setup and actual noise levels. That the information decreases with q-space radius is a consequence of the diminishing average signal energy present for all three distributions (and for all reasonable other distributions).

\section{Results}

The Allsorts distribution: Figure 2 shows the result of the estimated q-space metric for the Allsorts distribution. The lilac colored iso-surfaces show the 3D q-space locations where the information gain from a second sample, given a first sample in the center (yellow), reaches $\Delta I=2$ bits. Results for five different radii $(0,0.25,0.5,0.75$ and 1.0$)$ of the first sample are shown. The radii were chosen to highlight the typical information gain behaviors that will be present in different parts of q-space. Since the setup is rotationally invariant the results will be the same along any axis through the origin. The upper plot shows iso-surfaces along four different directions in one octant of q-space and is intended to demonstrate that the metric is rotation invariant. The lower part of figure 2 shows the five results obtained for the initial point located at different positions on the $\mathrm{x}$-axis. A short summary of the situation at different $\mathrm{q}$-space radii, $\mathrm{r}$, is given below:

- At $\mathbf{r}=\mathbf{0}$ : The second sample must be moved quite far from the first to gain more information, i.e. very sparse sampling is needed, a sample at $q=0$ picks up most of the information available at the center.

- At $\mathbf{r}=\mathbf{0 . 2 5}$ : The information gain now quickly increases in an approximately isotropic fashion when the second location is moved away from the first. This indicates that a relatively dense sampling is preferable here.

- At $\mathbf{r}=\mathbf{0 . 5}$ : The situation resembles the previous one but a slight anisotropy of the 2-bit iso-surface can be noted. The second sample has to be moved further in the radial direction than in an angular direction to give the same information gain.

- At $\mathbf{r}=\mathbf{0 . 7 5}$ : The anisotropy is becoming more pronounced indicating that moving the second location in the angular direction is clearly preferable to 

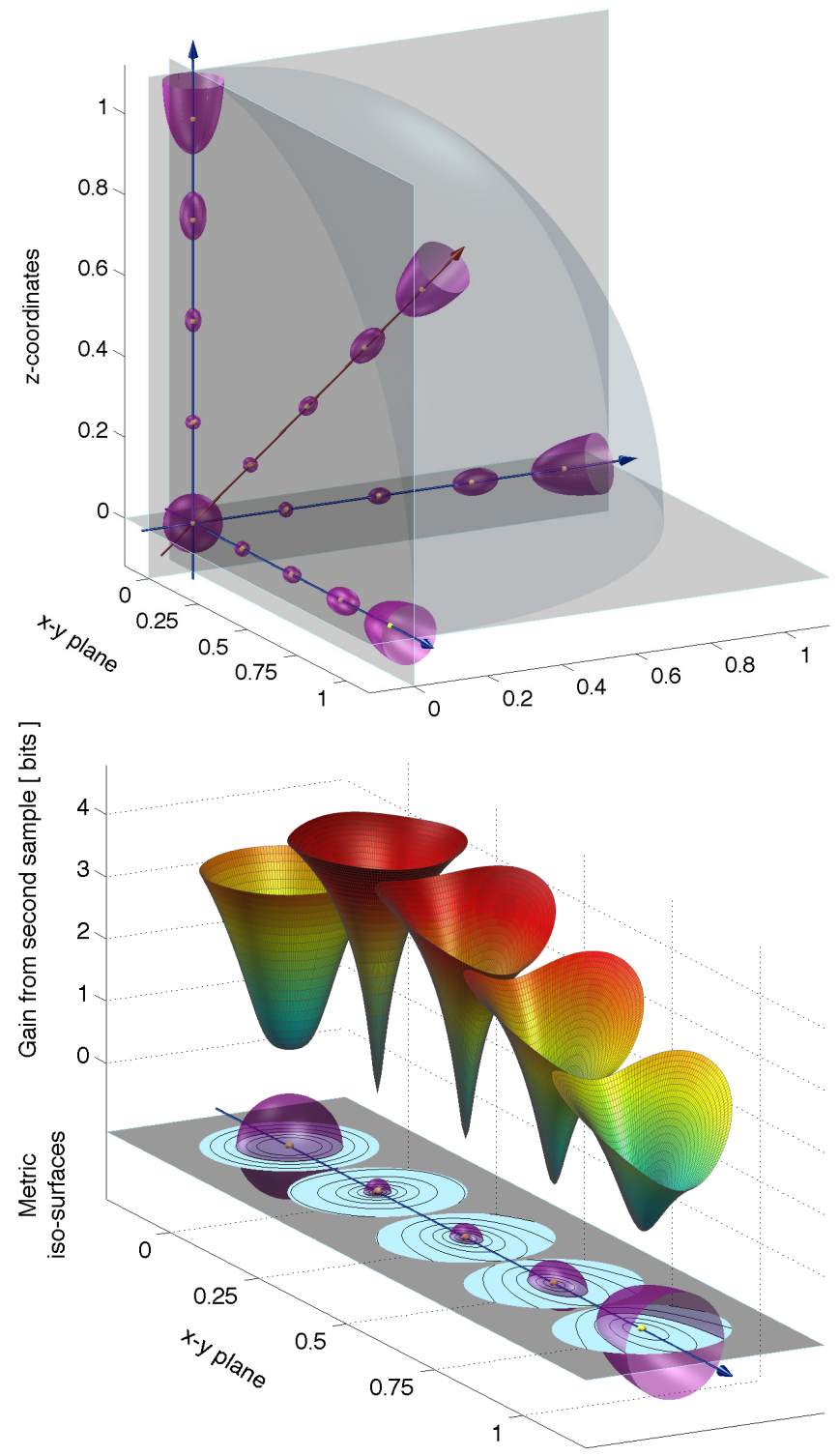

Fig. 2. Figure showing the result of the estimated q-space metric for the Allsorts distribution. The lilac colored iso-surfaces show where the information gain from a second sample, given a first sample in the center (yellow), reaches $\Delta I=2$ bits. The figure shows the results for five different radii of the first sample. The upper plot shows one octant of q-space and is intended to demonstrate that the metric is rotation invariant. The lower plot shows only the cases where the first sample is on the x-axes. The multi colored surfaces show the information gain when moving the second sample away from the first, the gain in bits is indicated by the numbers 1-4 on the upper part of the $\mathrm{y}$-axis. The iso-contours on the $\mathrm{x}$-y plane below are drawn for every $0.5 \mathrm{bits}$. 

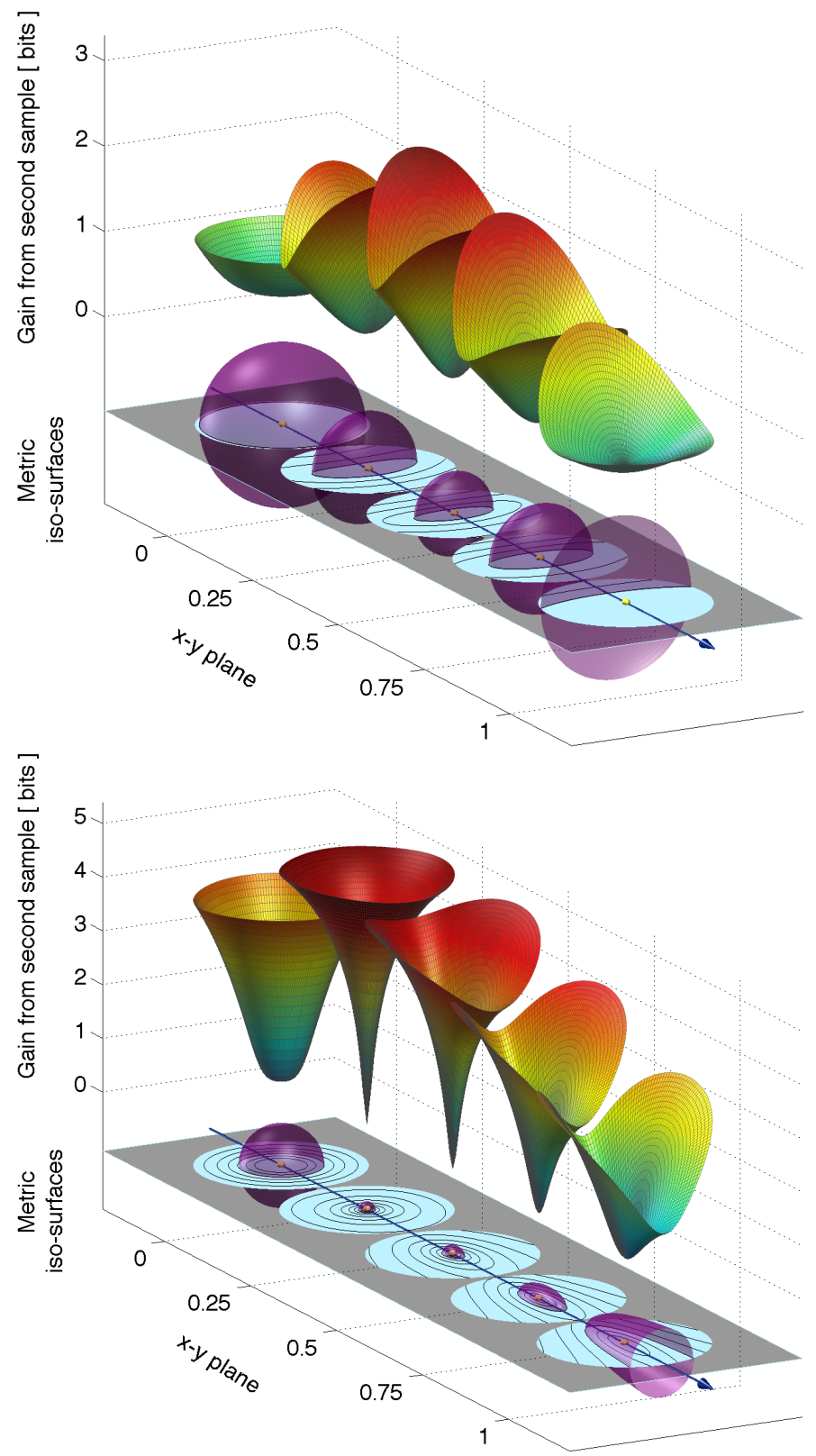

Fig. 3. Figure demonstrating the estimated q-space metric dependence on the propagator distribution. The upper plot shows the result for the Round distribution. The lilac colored iso-surfaces show where the information gain from a second sample, given a first sample in the center (yellow), reaches $\Delta I=1$ bit. The lower plot shows the result for the Stick distribution. Here the iso-surfaces are drawn at $\Delta I=2$ bits. For both plots the iso-contour line are drawn 0.5 bits apart. 
a change in radius. The volume enclosed by the iso-surface is also larger indicating that a less dense sampling is needed. The sampling distance in the radial direction should be lower than that in the angular direction.

- At $\mathbf{r}=1.0$ : The iso-surface now takes the shape of a cone implying that the information gain is largest when moving simultaneously inwards and angularly. This is due to that the SNR at this radius quickly decreases with increasing radius due to loss of signal strength.

The Round and Stick Distributions: By studying distribution that are individually more uniform and 'removed' from the Allsorts distribution in two different ways further insights can be gained. The plot at the top of figure 3 shows the results when using the Round distribution and below the results from using the Stick distribution is plotted. Comparing the two results the following can be noted:

- At $\mathbf{r}=\mathbf{0}$ : In both cases the second sample still must be moved relatively far from the first to gain more information. This effect is very pronounced for the Round distribution and the plots indicate that the second sample most be moved roughly four times as far as for the Stick distribution to gain the same amount of information. (Note that the iso-surfaces are drawn at different levels, $\Delta I=1$ bit and $\Delta I=2$ bits.)

- At $\mathbf{r}=\mathbf{0 . 2 5}$ : Here the difference between the two distribution is even bigger. For the Round distribution very little is gained by by displacing the second sample angularly, the preferred displacement direction is clearly radial. In contrast the information gain increases very quickly in an isotropic fashion for the Stick distribution.

- At $\mathbf{r}=\mathbf{0 . 5}$ and $\mathbf{r}=\mathbf{0 . 7 5}$ : The situation resembles the previous one but an increasing anisotropy of the 2-bit iso-surface can be noted for the Stick distribution. The second sample has to be moved further in the radial direction than in an angular direction to give the same information gain. A common feature is that the information gain vs displacement distance is decreasing for both distributions with increasing q-space radius.

- At $\mathbf{r}=1.0$ : The Stick distribution iso-surface now takes the shape of an open cone implying that the information gain is largest when moving simultaneously inwards and angularly. For the Round distribution the iso-surface almost becomes a plane. This indicates that we are close to a radius were higher information gain can only be obtained by moving towards the center, we are approaching the information 'edge' of q-space.

A general difference that is globally present is that it that the average information gain from a second sample is much lower for the Round distribution than for the Stick distribution. This is, most likely, a consequence of that the former distribution has a lower overall variability, i.e. lower entropy.

\section{Discussion and Conclusion}

Although the interpretation of the results may be accordance with the gut feeling of some experienced researchers in the field we believe that our analysis provides 
a novel view allowing a quantification of said feeling. Our analysis also stresses the fact that the actual average propagator distribution in the targeted tissue has a major effect on what is the optimal q-space sampling strategy. In many cases tissue models can be employed and educated guesses can be made regarding the average propagator distribution present. In such cases the presented analysis can, for example, be used to find parameters for the 3D q-space sample distribution scheme described in [10. In this way full q-space sampling, optimal with respect to a given expected distribution of diffusion propagators can be produced. This will also allow tuning of q-space distributions to maximize resolution for targeted tissue features.

Acknowledgement. The authors acknowledge the Swedish Research Council grants 2011-5176, 2012-3682, 2007-8632 (CADICS Linneaus research environment) and NIH grants R01MH074794, P41RR013218, and P41EB015902.

\section{References}

1. Jones, D.K., Simmons, A., Williams, S.C.R., Horsfield, M.A.: Non-invasive assessment of axonal fiber connectivity in the human brain via diffusion tensor MRI. Magn. Reson. Med. 42, 37-41 (1999)

2. Assaf, Y., Freidlin, R.Z., Rohde, G.K., Basser, P.J.: New modeling and experimental framework to characterize hindered and restricted water diffusion in brain white matter. Magn. Reson. Med. 52(5), 965-978 (2004)

3. Wu, Y.C., Alexander, A.: Hybrid diffusion imaging. Neuroimage 36(3), 617-629 (2007)

4. Alexander, D.C.: A general framework for experiment design in diffusion MRI and its application in measuring direct tissue-microstructure features. Magn. Reson. Med. 60(2), 439-448 (2008)

5. Merlet, S., Caruyer, E., Deriche, R.: Impact of radial and angular sampling on multiple shells acquisition in diffusion MRI. Med. Image Comput. Comput. Assist. Interv. 14(Pt. 2), 116-123 (2011); Eds: T. Peters, G. Fichtinger, A. Martel

6. Caruyer, E., Cheng, J., Lenglet, C., Sapiro, G., Jiang, T., Deriche, R.: Optimal Design of Multiple Q-shells experiments for Diffusion MRI. In: MICCAI Workshop CDMRI 2011 (2011)

7. Ye, W., Portnoy, S., Entezari, A., Blackband, S.J., Vemuri, B.C.: An Efficient Interlaced Multi-shell Sampling Scheme for Reconstruction of Diffusion Propagators. IEEE Trans. Med. Imaging 31(5), 1043-1050 (2012)

8. Westin, C.F., Pasternak, O., Knutsson, H.: Rotationally invariant gradient schemes for diffusion MRI. In: Proc. of the ISMRM Annual Meeting (ISMRM 2012), p. 3537 (2012)

9. Scherrer, B., Warfield, S.K.: Parametric Representation of Multiple White Matter Fascicles from Cube and Sphere Diffusion MRI. PLoS One 7(11), 1-20 (2012)

10. Knutsson, H., Westin, C.-F.: Charged Containers for Optimal 3D Q-space Sampling. In: ISMRM 2013 (2013)

11. Shannon, C.: Communication in the Presence of Noise. Proceedings of the IRE 37(1), 10-21 (1949)

12. Rao, C.R.: Bull. Calcutta Math. Soc. 37 (1945)

13. Pires, C.A.L., Perdigo, R.A.P.: Minimum Mutual Information and Non-Gaussianity Through the Maximum Entropy Method: Theory and Properties. Entropy 14, 1103-1126 (2012); Ed: K. H. Knuth 